\title{
Nonlinear Dynamics of International Gold Prices: Conditional Heteroskedasticity or Chaos?
}

\author{
Xiaowei HUANG \\ The School of Banking and Finance, University of International Business and Economics, Beijing \\ 100029, China \\ E-mail: xwhuang@uibe.edu.cn \\ Mei YU \\ The School of Banking and Finance, University of International Business and Economics, Beijing \\ 100029, China \\ E-mail:yumei@uibe.edu.cn \\ Chengwei BAN \\ Guanghua School of Management, Peking University, Beijing 100871, China \\ E-mail: banchengwei@gmail.com
}

\begin{abstract}
Taking the special nonlinear characteristics of the domestic and international gold price into account, this paper systematically analyzed its nonlinearity by the methods of BDS test, R/S analysis and improved largest Lyapunov exponent. We find three main results: (1) ARMA-GARCH model could adequately explain the linear and nonlinear dependence of gold price series; (2) long-memory does not exist anymore in price series explained by ARMA-GARCH model; (3) chaos phenomenon which is sensitive to the initial value does not exist either in the residuals of regression model. Therefore, we believe that the nonlinearity of gold price is mainly characterized in conditional heteroscedasticity rather than chaos.
\end{abstract}

Keywords heteroscedasticity; chaos; BDS test; R/S analysis; Lyapunov exponent

\section{Introduction}

With the fluctuations of the global economy and the depression of expectation in recent years, the international price of gold reached one after another peak. As a symbol of the precious metal prices, the nonlinear characteristics of the changes of the prices of gold reflect the characteristics of the changes of the macroeconomic. Whether the nonlinearity of the gold price is chaotic or not affects the judgment of the characteristics of the macroeconomic. Many scholars have found nonlinear features in commercial price such as conditional heteroscedasticity. But it is hard to observe directly whether there is chaos behind the superficial price fluctuation.

The characteristic of the nonlinearity of the price of gold is essential. If the nonlinear fluctuation shows only in conditional heteroscedasticity, it is safe and proper to follow the EMH

Received March 4, 2014, accepted April 29, 2014

Supported by National Social Science Foundation of China (No. 12CGJ027) 
framework to analyze the macroeconomic and the financial market, and it is still reasonable to hold the points that the yield follows a martingale process and the prices of the assets can be predicted. But if the nonlinearity of the price is chaos, suggesting that it is a deterministic lowdimension dynamic system, the randomness of asset prices then comes from their sensitivity to the initial state mostly rather than the turbulence outside. The abnormal yield produced by a chaotic dynamic system happens to explain the extremely negative yield of the capital market, such as the stock disaster in 1987, the so-called "six sigma event". If it is really chaotic, the supervisor should change the methods they use to regulate the market.

The common tests for randomness cannot distinguish the chaos in the financial time series data with so much noise. The most effective way nowadays for testing chaos phenomenon are the followings: BDS test, R/S test and the largest Lyapunov test. The three methods are not contradictory, they are testing the chaos phenomenon from different perspectives, that is, nonlinear dependence, long memory and sensitivity of initial conditions. Firstly, BDS test is a nonparametric test designed to test if there is nonlinear dependence, whose result is sensitive to the choice of embedding dimension and proximity parameters. Secondly, R/S test is capable of testing the long-term memory in the data, judging whether the reverting phenomenon is negative or positive and finding the average period of the data. Thirdly, largest Lyapunov exponent describes the sensitivity of the motion to its initial conditions quantitatively and reflecting its chaotic characteristic.

Most of the methods testing chaos have some relationship with correlation integral. Grassberger and Pocaccia ${ }^{[5]}$ proposed the correlation integral and correlation dimension test to test the dimension of a chaotic system. Since the existence of nonlinear dependence is a prerequisite of the existence of chaos, Brock, Dechert and Scheinkman proposed the BDS test based on the correlation integral to test the nonlinear dependence of financial series data. Similarly, with a basis of the correlation integral, Kim et al. ${ }^{[7]}$ proposed CC phase space reconstruction algorithm, which, relying on Takens' theorem, is the basis for calculating Lyapunov exponents. The largest Lyapunov exponent gives a chaotic system a quantitative description on its sensitivity to the initial state of the system. Aimed at the largest Lyapunov exponent, Rostentein et al. ${ }^{[12]}$ proposed the small-amount-data algorithm that is not only simple and direct but also more accurate than the previous algorithms.

Frank and Stengos ${ }^{[4]}$ estimated the correlation dimensions and Kolmogorov entropy of gold and silver spot prices, finding chaotic characteristics. DeCoster et al. ${ }^{[3]}$ calculated the correlation dimensions of the futures of sugar, silver, copper and coffee, in which evidence for the existence of chaos had been found. By calculating correlation integral and carrying out BDS test, Yang and Brorsen ${ }^{[15]}$ analyzed prices of several future products and pointed out that chaos existed in prices of commodity futures. Arjun et al. (2001), however, found nonlinear dependence instead of chaos existed in series by calculating correlation integral and Kolmogorov entropy and testing residuals of ARMA-GARCH model. Also, Andres et al. (2002) discovered through BDS and Kaplan tests that nonlinear characteristics existed in foreign exchange rates series. Wang et al. ${ }^{[13]}$ calculated the correlation dimensions $d$ of Shanghai Composite Index using G-P algorithm, and Lyapunov exponent using Wolf method and reached a conclusion that chaos existed in Chinese stock market. 
By reviewing the recent literatures, we found that direct test of nonlinearity without delinearizing series, i.e. filtering the series by ARMA model, would lead to a conclusion that chaos existed in series. The reason for the conclusion is that current BDS and R/S analytical method both rejected i.i.d. ${ }^{1}$ assumption in testing linear, nonlinear and chaos characteristics. Besides, the adoption of low order $\mathrm{ARCH}$ model $^{2}$ in filtering the nonlinear characteristics of series frequently leads to wrong conclusion that chaos exists in series. So far there is no widely accepted conclusion on whether nonlinear characteristics of financial time series are results of low dimension chaos or conditional heteroscedasticity. Considering the robustness of the empirical results and the principle of "test test and test", we determinate the nonlinear characteristics of gold price series by carrying out BDS test and R/S analysis and calculating largest Lyapunov exponent respectively in these papers.

\section{$2 \quad$ BDS tests of gold prices in home and abroad}

BDS test is usually used to detect nonlinear dependence structure of series, popular among researches because of its non-substitutability. Our BDS test idea is as follows:

- Pre-process data by converting price series $\left\{X_{N}\right\}$ to $\log$ return series $\left\{R_{N-1}\right\}$, carry out descriptive statistical analysis and test whether the series are weak stationary by ADF unit root tests.

- Test the hypothesis that return series are i.i.d. by BDS test. The rejection of i.i.d. hypothesis means linear of nonlinear dependence exists in series.

- Eliminate the linear dependence of series. We choose BIC information criteria and parameters t-test to choose the proper ARMA model for each series thus to filter the linear structure, followed by BDS test on residuals. The failure of BDS test illustrated nonlinear dependence of series, thus we should consider whether it results from characteristics in various time periods.

- Due to the long time span of data collection ${ }^{3}$, it is possible that characteristics of series in different time periods cause series' failure to be i.i.d.. In order to exclude this possibility, we divide return series into two sample intervals of the same length, filter respectively with optimal linear model and carry out BDS tests on their residuals. If test results are significantly different from those of entire population, then the reason why population fails BDS test might be the structural change of population series; if test results are not distinct different, then time-varying variance should be considered as a cause.

- Exclude the time-varying variance in series. Xie and Yang ${ }^{[14]}$ standardized series with standard error in current month, paving the way for such a practice. More specifically, we use GARCH model to filter the time-varying variance, and then choose the optimal linear model under BIC information criteria. After excluding the effects of time-varying

\footnotetext{
${ }^{1}$ In this paper i.i.d. refers to Independent Identically Distributed.

${ }^{2}$ For example, $\operatorname{ARCH}(1), \operatorname{ARCH}(2)$.

${ }^{3}$ oversea data of 35 -year and domestic data of 9 -year.
} 
variance, we conduct BDS test again. If BDS test is passed, we can conclude that ARMAGARCH model can explain the characteristics of series effectively, and that chaos doesn't exist in series. Otherwise nonlinear structure such as chaotic systems exists in series, which can not be detected by AMRA-GARCH model.

\subsection{BDS test}

BDS test is based on a basic idea that, if a series $\left\{X_{n}\right\}$ is i.i.d., then $\forall i \neq j, \forall \varepsilon, \forall m$, we have

$$
\begin{aligned}
& P_{2}=P\left(\left|X_{i}-X_{j}\right| \leq \varepsilon,\left|X_{i-1}-X_{j-1}\right| \leq \varepsilon\right)=\left[P\left(\left|X_{i}-X_{j}\right| \leq \varepsilon\right)\right]^{2} \\
P_{m} & =P\left(\left|X_{i}-X_{j}\right| \leq \varepsilon,\left|X_{i-1}-X_{j-1}\right| \leq \varepsilon, \cdots,\left|X_{i-m+1}-X_{j-m+1}\right| \leq \varepsilon\right) \\
& =\left[P\left(\left|X_{i}-X_{j}\right| \leq \varepsilon\right)\right]^{m}
\end{aligned}
$$

where $\varepsilon$ shows the approaching level (often called approach parameter) of status in the series, $m$ shows the dimension of reconstructed phase space (often called embedding dimension). The equations above are established if and approximately only if time series $\left\{X_{n}\right\}$ is i.i.d.. Thus by selecting any of the equations as null hypothesis we can test whether $\left\{X_{n}\right\}$ is i.i.d.. In practice, Brock et al. (1987) calculated correlation integral $C_{m, n}(\varepsilon)$ and $P_{m}$ acquired, based on which later scholars made much generalization and improvements. In this paper we choose Kanzler's ${ }^{6]}$ method, a practical generalization in 1999 based on Brock's achievements that can be described as follows. First we define indicative function

$$
I_{\varepsilon}\left(X_{i}, X_{j}\right)= \begin{cases}1, & \left|X_{i}-X_{j}\right| \leq \varepsilon \\ 0, & \left|X_{i}-X_{j}\right|>\varepsilon\end{cases}
$$

For finite time series $\left\{X_{n}\right\}$, proper proximity parameter $\varepsilon$ and embedding dimension $m$, we define correlation integral revised by Kanzler as follows:

$$
C_{m, n}(\varepsilon)=\frac{2}{(n-m+1)(n-m)} \sum_{s=m}^{n} \sum_{t=s+1}^{n} \prod_{j=0}^{m-1} I_{\varepsilon}\left(X_{s-j}, X_{t-j}\right)
$$

If $\left\{X_{n}\right\} \sim$ i.i.d., correlation integral $C_{m, n}(\varepsilon)$ approximately equals to $\left[C_{1, n-m+1}(\varepsilon)\right]^{m}$ and we could use $\left[C_{1, n-m+1}(\varepsilon)\right]^{m}$ to estimate the mean of $C_{m, n}(\varepsilon)$. After standardizing $C_{m, n}(\varepsilon)$ we could get statistic $W$ :

$$
W_{m, n}(\varepsilon)=\sqrt{n-m+1} \frac{C_{m, n}(\varepsilon)-\left[C_{1, n-m+1}(\varepsilon)\right]^{m}}{\sigma_{m, n}(\varepsilon)}
$$

where

$$
\begin{aligned}
\hat{\sigma}_{m, n}(\varepsilon)=4\left\{k^{m}+\right. & \left.2 \sum_{j=1}^{m-1} k^{m-j} C_{1, n}(\varepsilon)^{2 j}+(m-1)^{2} C_{1, n}(\varepsilon)^{2 m}-m^{2} k C_{1, n}(\varepsilon)^{2 m-2}\right\} \\
k_{n}(\varepsilon)= & \frac{2}{n(n-1)(n-2)} \sum_{t=1}^{n} \sum_{s=t+1}^{n} \sum_{r=s+1}^{n}\left\{I_{\varepsilon}\left(X_{t}, X_{s}\right) I_{\varepsilon}\left(X_{s}, X_{r}\right)\right. \\
& \left.+I_{\varepsilon}\left(X_{t}, X_{r}\right) I_{\varepsilon}\left(X_{r}, X_{s}\right)+I_{\varepsilon}\left(X_{s}, X_{t}\right) I_{\varepsilon}\left(X_{t}, X_{r}\right)\right\}
\end{aligned}
$$

Brock et al. ${ }^{[2]}$ demonstrated that under the assumption of $\left\{X_{n}\right\} \sim$ i.i.d., the limiting distribution of $W_{m, n}(\varepsilon)$ is standard norm distribution. When $\frac{n}{m} \geq 200$, quantile of standard norm distribution can be used as judgment criteria. 
Two important parameters should be set before BDS test: proximity parameter $\varepsilon$ and embedding dimension $m$ (see Table 1 for a summary on parameter choice). In theory, we need only to satisfy $0<\varepsilon<\max \left(X_{n}\right)-\min \left(X_{n}\right)$ to choose proximity parameter $\varepsilon$. However, Kočenda ${ }^{[8]}$ pointed out that the result of BDS test is quite sensitive to the choice of $\varepsilon$, and during practice we also found that as long as slight difference exists in proximity parameter, the calculated correlation integral varies greatly and thus causes the result of BDS test to be entirely different. In actual testing practice, it often occurs that the results contradict each other under different proximity parameter $\varepsilon$. Improper proximity parameter could incorrectly measure the closeness between data, which is the same as using a too-long or too-short ruler to measure the length of an object, causing relatively large errors.

Table 1 A summary on worldwide scholar's choice of proximity parameter

\begin{tabular}{lll}
\hline Year & Scholar Name & $\varepsilon$ (multiple of series standard error $\sigma$ ) \\
\hline 1992 & Rothman & $0.50,1.00,1.25,1.50,2.00$ \\
1993 & Kugler and Lenz & 1 \\
1993 & Hsieh & $0.50,1.00,1.50,2.00$ \\
1993 & Brock, Hsieh, and LeBaron & $0.50,0.75,1.00,1.25,1.50$ \\
1996 & Cecen and Erkal & 0.5 \\
1996 & de Lima & $1.00,1.25$ \\
1996 & Chappell, Padmore, and Ellis & $0.40,0.625,1.00,1.60$ \\
1997 & Serletis and Gogas & $0.50,1.00,1.50,2.00$ \\
2000 & Brooks and Henry & $1.00,1.50$ \\
2000 & Aguirre and Aguirre & $0.65,0.70,0.75,0.80,0.85,0.90$ \\
2002 & Chen and Kuan & $0.75,1.00$ \\
2003 & Panagiotidis & $0.50,1.00,2.00$ \\
2004 & Kočenda and Briatka & 0.6 to 1.9 \\
\hline
\end{tabular}

Through empirical study, Kanzler discovered that under the condition of smaller embedding dimensions $m$, BDS statistic $W_{m, n}\left(\varepsilon^{*}\right)$ is optimal if we choose proximity parameter $\varepsilon$ which makes single dimension correlation integral $C_{1, n}(\varepsilon)$ near 0.7 . The algorithm's complexity in time and space is rather high and, as Jorge et al. (2002) mentioned, computer capacity in the past could hardly satisfy its requirement. However, with the significant improvement of computer performance and algorithm, optimizing proximity parameter $\varepsilon$ becomes feasible and easy. In this paper we adopt Kanzler's method, for each embedding dimension $m$, choose an optimal proximity parameter $\varepsilon$ on conditions that single dimension correlation integral $C_{1, n}(\varepsilon)$ is fixed to 0.7, and thus obtain the optimal BDS statistic $W_{m, n}\left(\varepsilon^{*}\right)$. In terms of choosing the embedding dimension, Brock et al. ${ }^{[2]}$ suggested that, on the premise of ensuring $\frac{n}{m} \geq 200$, choose $m$ between 2 and 10; Barnett et al. ${ }^{[1]}$ suggested $m$ not exceed 8 . In this paper, based on conservative principles, we choose all embedding dimension $m$ between 2 and 10 to satisfy $\frac{n}{m} \geq 200$.

\subsection{Data sample selection}

During the century before the end of The Bretton Woods System, gold underlies the currencies of the world. It makes little sense to study the 'fixed' gold price. In 1997 America 
ended the convertibility of gold into US dollars, terminating the gold standard that had lasted for more than a century. Since then, gold has faded from the currency scene and evolved into a commodity with price attribute. London Gold Market and COMEX, with their long history, have been the largest spots and futures market, and therefore data from the two exchanges are representative. We choose daily London Gold Fixing in London Gold Market and closing price of dominant futures of gold in COMEX from 2nd Jan. 1975 to 19th Apr. 2011 ${ }^{4}$. For domestic data, we choose daily closing price of spot Gold AU9995 in Shanghai Gold Exchange from 31st Oct. 2002 to 20th Apr. 2011 ${ }^{5}$. After the founding of New China, the purchasing, marketing and pricing of gold has been monopolized. The trading price of gold hasn't come out until the opening of Shanghai Gold Exchange in Oct. 2002. Gold AU9995 indicates gold with purity above $99.95 \%$. Its spot trading has been active, and it has been the settlement for gold futures in major future exchanges home and abroad. Consider that Chinese future market of gold wasn't formally formed until 2008, the data volume is limited, and thus we don't use domestic gold future data.

In this paper we converted gold prices of London spot, COMEX futures and Shanghai futures into logarithmic return series, conducting unit root test and obtained the stationary, descriptive statistics and unit-root test results of series (see Table 2 and Table 3).

Table 2 Descriptive statistics and normality test

\begin{tabular}{lcccccc}
\hline & Mean & SD & Skewness & Kurtosis & JB statistic & P-value \\
\hline London spot price & 0.0002 & 0.0126 & 0.0027 & 14.17 & 47693.79 & 0.0000 \\
COMEX future price & 0.0002 & 0.0126 & 0.0084 & 10.07 & 18845.71 & 0.0000 \\
Shanghai spot price & 0.00065 & 0.0113 & -0.2563 & 9.221 & 3369.85 & 0.0000 \\
\hline
\end{tabular}

Table 3 Unit root test

\begin{tabular}{lccccc}
\hline & \multicolumn{2}{c}{ ADF test } & & \multicolumn{2}{c}{ Philips-Perron test } \\
\cline { 2 - 3 } \cline { 5 - 6 } & T statistic & P value & & Adjusted t statistic & P value \\
\hline London spot price & -14.93347 & 0.0000 & & -100.0707 & 0.0001 \\
COMEX future price & -15.30907 & 0.0000 & & -96.68592 & 0.0001 \\
Shanghai spot price & -31.0917 & 0.0000 & & -47.94408 & 0.0001 \\
\hline
\end{tabular}

\subsection{Results of BDS tests}

\subsubsection{BDS test of the original series}

In order to test the existence of dependence in original series, we first carry out BDS test on original return series. Here we choose the proximity parameter $\varepsilon^{*}$ which makes the single dimensional correlation integral $C_{1, n}(\varepsilon)$ approximate 0.7 , and we choose the embedding dimension $m$ between 2 to 10. BDS test results of original series are in Table 4 . As the table shows, test results in all embedding dimensions reject in $1 \%$ significance level the hypothesis that original return series is i.i.d., but we cannot decide the existence of nonlinear dependence in the series.

\footnotetext{
${ }^{4}$ data source: Wind financial database.

${ }^{5}$ data source: Resset financial research database.
} 
Table 4 BDS test of original series

\begin{tabular}{lccccccccc}
\hline$m$ & 2 & 3 & 4 & 5 & 6 & 7 & 8 & 9 & 10 \\
\hline London spot price & 25.8 & 32.3 & 37.4 & 42.1 & 47.1 & 52.2 & 58.1 & 64.7 & 72.5 \\
COMEX future price & 22.9 & 28.2 & 31.9 & 35.2 & 38.8 & 42.9 & 47.6 & 52.8 & 59.0 \\
Shanghai spot price & 9.32 & 12.1 & 14.1 & 15.8 & 17.4 & 19.1 & 21.2 & 23.5 & 25.9 \\
\hline
\end{tabular}

Note: All test results rejected the null hypothesis of i.i.d..

\subsubsection{BDS test after linear model filtration}

Based on the results that hypothesis of i.i.d. are all rejected in original series, we choose the optimal ARMA model under BIC information criteria and t-test of auto-regression parameters to filter and eliminate the linear dependence of return series, and then test the residuals of ARMA model to decide whether nonlinear dependence existed in series. For London spot return series we choose $\operatorname{ARMA}(3,3)$ as linear model, for COMEX future return $\operatorname{ARMA}(3,3)$, and for Shanghai spot return MA(2)(see Table 5 for detailed results). After eliminating the linear dependence with optimal linear model, we test the residuals of the three return series respectively (see Table 6 for test results).

Table 5 Parameters in ARMA-GARCH model of London gold spots

\begin{tabular}{cccc}
\hline \multicolumn{2}{c}{ Mean Equation } & \multicolumn{2}{c}{ Variance Equation } \\
\hline $\mathrm{c}$ & $1.50 \times 10^{-5}$ & $\omega$ & $7.13 \times 10^{-7 * * *}$ \\
& $(0.175055)$ & & $(10.69556)$ \\
$\theta_{1}$ & $-0.031518^{* *}$ & $\alpha_{1}$ & $0.083008^{* * *}$ \\
& $(-2.892106)$ & & $(32.95886)$ \\
$\theta_{2}$ & $0.017221^{*}$ & $\beta_{1}$ & $0.917649^{* * *}$ \\
& $(1.657169)$ & & $(405.1998)$ \\
$\theta_{3}$ & $0.016047^{*}$ & & \\
& $(1.479584)$ & & -6.378732 \\
R-squared & 0.002467 & Durbin-Watson stat & -6.373296 \\
Loglikelihood & 29263.06 & Akaike info criterion & \\
F-statistic & 3.777334 & Schwarz criterion & \\
\hline
\end{tabular}

Table 6 BDS statistic for residuals of ARMA model

\begin{tabular}{lccccccccc}
\hline$m$ & 2 & 3 & 4 & 5 & 6 & 7 & 8 & 9 & 10 \\
\hline London spot price & 24.9 & 31.5 & 36.6 & 42.2 & 46.1 & 51.1 & 56.7 & 36.1 & 70.5 \\
COMEX future price & 22.6 & 27.8 & 31.5 & 34.7 & 38.1 & 42.0 & 46.6 & 51.7 & 57.7 \\
Shanghai spot price & 9.27 & 11.9 & 13.9 & 15.5 & 17.2 & 18.9 & 20.9 & 32.2 & 25.7 \\
\hline \multicolumn{2}{c}{ Note: All test results rejected the null hypothesis of i.i.d.. } \\
\hline \multicolumn{7}{c}{}
\end{tabular}

The ARMA model residuals of London spot return, COMEX future return and Shanghai spot return all reject the i.i.d. hypothesis in $1 \%$ significance level. This indicates that ARMA model cannot effectively explain the dependent characteristic, which is the nonlinear dependence, in gold return series. To further determine whether the cause is the characteristics that 
vary with different time period, we divide gold return series into two parts of the same length, and filter with proper ARMA model respectively. Here we filter with ARMA model series from London, New York and Shanghai, which add up to 6 subsample intervals, and conduct BDS test on obtained residuals. In view of the short length of Shanghai spot return series, the embedding dimension $m$ were chose between 2 to 6 (see detailed results in Table 7).

Table 7 BDS tests of ARMA model residuals on subsample intervals

\begin{tabular}{|c|c|c|c|c|c|c|}
\hline \multirow{3}{*}{$m$} & \multicolumn{2}{|c|}{ London spot price } & \multicolumn{2}{|c|}{ COMEX future price } & \multicolumn{2}{|c|}{ Shanghai spot price } \\
\hline & 1975-1993 & 1993-2011 & 1975-1993 & 1993-2011 & $2002-2007$ & 2007-2011 \\
\hline & $\operatorname{ARMA}(3,3)$ & $\operatorname{ARMA}(3,3)$ & $\operatorname{ARMA}(3,3)$ & $\operatorname{ARMA}(2,2)$ & $\operatorname{ARMA}(4,4)$ & $\operatorname{ARMA}(3,1)$ \\
\hline 2 & 20.99 & 10.82 & 18.45 & 9.90 & 7.30 & 4.68 \\
\hline 3 & 25.88 & 15.14 & 21.71 & 13.73 & 9.43 & 5.68 \\
\hline 4 & 29.86 & 18.33 & 24.35 & 16.34 & 10.54 & 7.09 \\
\hline 5 & 33.27 & 21.31 & 26.68 & 18.69 & 11.31 & 8.10 \\
\hline 6 & 37.04 & 24.30 & 29.27 & 21.17 & 12.01 & 9.13 \\
\hline 7 & 40.93 & 27.38 & 32.17 & 23.95 & & \\
\hline 8 & 45.38 & 30.78 & 35.51 & 26.99 & & \\
\hline 9 & 50.30 & 34.53 & 39.11 & 30.36 & & \\
\hline 10 & 56.00 & 39.09 & 43.48 & 34.18 & & \\
\hline
\end{tabular}

Note: All test results rejected the null hypothesis of i.i.d..

The test results agree on different subsample intervals. Residuals of ARMA model all reject the i.i.d. hypothesis. Although BDS statistic varies with different intervals, the i.i.d. hypothesis are all rejected in minuscule significant level. According to this, we find that the nonlinear dependence in series is not caused by the different characteristic in different time period.

\subsubsection{Test after conditional heteroscedasticity model filtration}

Here we test whether the nonlinear dependence is caused by time-varying variance of the return series. We choose ARMA-GARCH model to eliminate the time-varying variance and obtain the MA(3)-GARCH(1,1) model for London spot, $\operatorname{ARMA}(5,5)-\operatorname{GARCH}(4,4)$ model for COMEX future and MA(2)-GARCH $(1,1)$ model for Shanghai spot, and then carry out BDS test on standardized residuals(see results in Table 8).

Table 8 BDS tests on residuals of fitting models

\begin{tabular}{lccccccccc}
\hline Subintervals & 2 & 3 & 4 & 5 & 6 & 7 & 8 & 9 & 10 \\
\hline London spot price & 0.45 & 0.26 & 0.61 & 0.92 & 1.34 & 1.47 & 1.56 & 1.53 & 1.63 \\
COMEX future price & -0.11 & -0.49 & -0.63 & -0.92 & -0.97 & -0.85 & -0.74 & -0.70 & -0.54 \\
Shanghai spot price & -0.33 & -0.24 & -0.34 & -0.15 & -0.14 & -0.18 & -0.15 & -0.09 & -0.09 \\
\hline
\end{tabular}

Note: all test results accepted the null hypothesis of i.i.d..

Almost all test results accept the i.i.d. hypothesis ${ }^{6}$, which showed the nonlinear dependence of gold return series could be fully described with GARCH model. In other words, from the

\footnotetext{
${ }^{6}$ Actually under higher embedding dimension $m$, the null hypothesis of BDS test is also accepted for regression residuals of both London spot and New York futures.
} 
aspects of BDS tests, nonlinear dependence beyond ARMA-GARCH model doesn't exist in the daily gold price series, i.e., chaos doesn't exist.

\section{$3 \mathrm{R} / \mathrm{S}$ analysis on gold price at home and abroad}

Hurst discovered the durability of water level when studying the Niles River and calculated Hurst index through rescaled range analysis, which quantified the characteristic. Peters ${ }^{[10,11]}$ pointed out that $\mathrm{R} / \mathrm{S}$ analysis was an effective tool in discriminating fractional noise (random sequence) and chaotic noise series, and could observe the series' average period of non-periodic cycle. An evidence for the existence of chaotic attractor is the characteristic of infinite period. As a result R/S analysis can be an effective judgment on the existence of chaos. Lo (1991) proposed revised R/S analysis method and can eliminate the disturbance of short-term memory and heteroscedasticity.

Here we hope to determine the nonlinear attributes of series by R/S analysis and we divide the empirical method into two aspects: On one hand, since the standardized residuals (hereinafter 'fitting model residuals') we use where the short-term memory and heteroscedasticity in series have been removed by ARMA-GARCH models ${ }^{7}$, we can employ directly the classic $\mathrm{R} / \mathrm{S}$ analysis. In this paper we employ $\mathrm{R} / \mathrm{S}$ analysis on original gold price series and fitting model residuals respectively, and by comparing these empirical study results we can determine the nonlinear attributes of series. On the other hand, according to the parameters in fitting models, we employ R/S analysis on a generated series, which uses random numbers as residuals and has same characteristics with the original series, and study the difference between two analysis results. No difference between them indicates that simple ARMA-GARCH model can generate same characteristics with original series, which means no characteristics beyond ARMA-GARCH model exist in original series.

\section{$3.1 \quad \mathrm{R} / \mathrm{S}$ analysis method}

For sequence $\left\{X_{n}\right\}, n=1,2, \cdots, N, \cdots, 2 N, \cdots, 3 N, \cdots$, the length for each subsequence is $N$. Here we divide the sequence to several nonintersecting $N$-length subsequences, and calculate the accumulative deviation for each subsequence

$$
D_{t, N}=\sum_{u=1}^{t}\left(X_{u}-M_{N}\right)
$$

where $M_{N}$ is mean of $X_{n}$. The difference between maximum and minimum cumulative deviation is regarded as the range of $N$-length subsequence of series $\left\{X_{n}\right\}$

$$
R_{N}=\operatorname{MAX}\left(X_{t, N}\right)-\operatorname{MIN}\left(X_{t, N}\right)
$$

To eliminate the effect of dimension and ensure the scale invariance of statistics, Hurst introduced rescaled range $\frac{R_{N}}{S_{N}}$, in which $S_{N}$ stood for the standard deviation of original series. Since for each appropriate $N$, a comparatively long sequence can be divided into several nonintersecting subsequences, here we use the mean of rescaled range $\frac{R_{N}}{S_{N}}$ as observation for $N$-length rescaled range. In order to ensure the uniformity of data in calculating rescaled range, we intercept a $T$-length sequence which is relatively long and contains more factors, and choose all

\footnotetext{
${ }^{7}$ Here ARMA model is identical to that used in BDS test.
} 
factors above 4 and below $\frac{T}{2}$ as length of subsequence, $N$.

Hurst discovered that rescaled range was a power function of observation time $N$, satisfying

$$
\frac{R_{N}}{S_{N}}=(a N)^{H}
$$

where $a$ is constant and $H$ is Hurst index. In practice we often convert the above equation to logarithmic form

$$
\ln \frac{R_{N}}{S_{N}}=H \ln N+\ln b
$$

For each observation time $N$, we obtain different rescaled range $\frac{R_{n}}{S_{n}}$, and get an estimation for Hurst index through LS regression.

There are three possible values for Hurst index: 1) $H=0.5$, which means the sequence is random and independent; 2) $0 \leq H<0.5$, which means the system is ergodic or antipersistant; 3) $0.5<H<1.00$, which means the sequence is persistence. In fact, since the length of sequences is finite, the Hurst index of randomly generated sequence with complete i.i.d. does not equal to 0.5. As a result, based on previous study results, Peters (1994) obtained the expectation of $\frac{R_{n}}{S_{n}}, E\left(\frac{R_{n}}{S_{n}}\right)$ under the assumption of i.i.d. ${ }^{8}$.

$$
E\left(\frac{R_{N}}{S_{N}}\right)=\frac{N-0.5}{N} \times\left(N \times \frac{\pi}{2}\right)^{-0.5} \times \sum_{r=1}^{N-1} \sqrt{\frac{N-r}{r}}
$$

Similarly, through LS regression we can obtain an estimation of Hurst index expectation. By comparing analysis results of time series $\frac{R_{n}}{S_{n}}$ with expectation $E\left(\frac{R_{n}}{S_{n}}\right)$ under i.i.d. assumption, we can discriminate the difference between long memory and i.i.d..

\subsection{Analysis results of $\mathrm{R} / \mathrm{S}$}

\subsection{1 $R / S$ analysis on empirical data}

We carry out R/S analysis on series of spot price in London and Shanghai ${ }^{9}$. In Figure 1, the steep curve is $\ln \left(\frac{R_{n}}{S_{n}}\right)$ of original return series and the gradual curve is the expectation of $\ln \left(\frac{R_{n}}{S_{n}}\right), \ln \left(E\left(\frac{R_{n}}{S_{n}}\right)\right)$ under i.i.d. assumption. It is obvious that for original gold price series, the

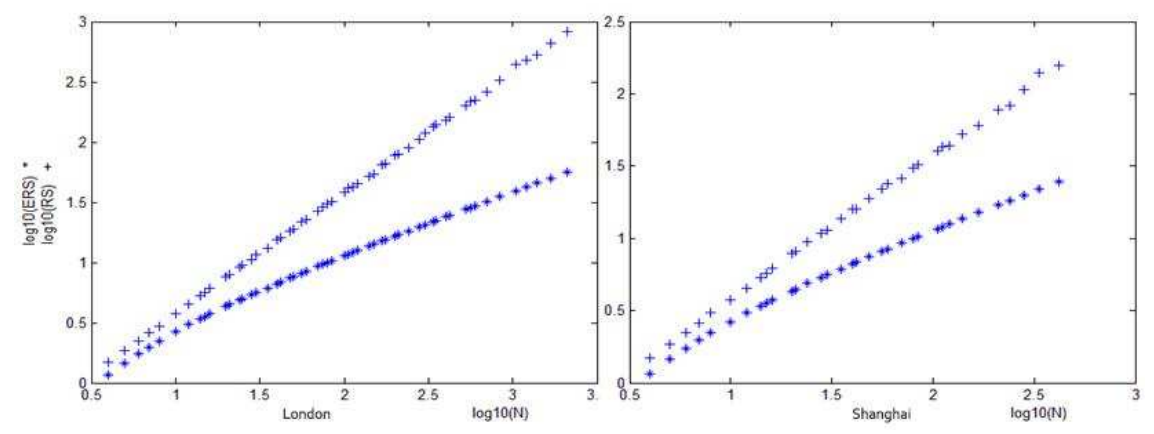

Figure $1 \mathrm{R} / \mathrm{S}$ analysis on original gold price data in London and Shanghai

\footnotetext{
${ }^{8}$ In this paper, $E\left(\frac{R_{n}}{S_{n}}\right)$ refers to the expected value of rescaled range when the series is i.i.d..

${ }^{9}$ We only show R/S analysis results of London spot and Shanghai spot here. New York futures shares similar results.
} 
slope of $\ln \left(\frac{R_{n}}{S_{n}}\right)$ to $\ln (N)$ approximates 1 , which is significantly different from the slope of $\ln \left(E\left(\frac{R_{n}}{S_{n}}\right)\right)$ to $\ln (N)$. This means that long memory exists in original series. Besides, the Hurst index result obtained from LS regression approximates 1 (see Table 9 for results), also supporting the conclusion. The long memory of original series might be the result of both ARMA structure and GARCH effect.

Table 9 Hurst index of gold data under different processing at home and abroad

\begin{tabular}{lcc}
\hline & Gold in London & Gold in Shanghai \\
\hline Hurst index of original price series & 1.0091 & 0.9997 \\
Hurst index of residuals of ARMA-GARCH model & 0.5921 & 0.6225 \\
Expectation of Hurst index under i.i.d. & 0.5897 & 0.6301 \\
\hline
\end{tabular}

However, after converting gold price series into logarithmic return series and filtering with ARMA-GARCH model, we obtain a significantly different result (see Figure 2 for details): No difference exists between fitting model residuals of gold price and expectations of i.i.d. series, which means that in the series, long-term memory beyond the ARMA-GARCH process does not exist in series. According to Fig. $2, \ln \left(\frac{R_{n}}{S_{n}}\right)$ overlaps $\ln \left(E\left(\frac{R_{n}}{S_{n}}\right)\right)$ in standardized residuals, and both slope approximates each other, which shows the absense of long-term memory. The result differs from Peters' due to two reasons. First, Peters employed R/S analysis directly on price series instead of return series. Second, Peters left out the effects of ARMA structure and conditional heteroscedasticity in series. Besides, we can see from the following table that the long-term memory of original price series is quite obvious, while that of return series is relatively weak. In fitting model residuals, long-term memory does not exist.

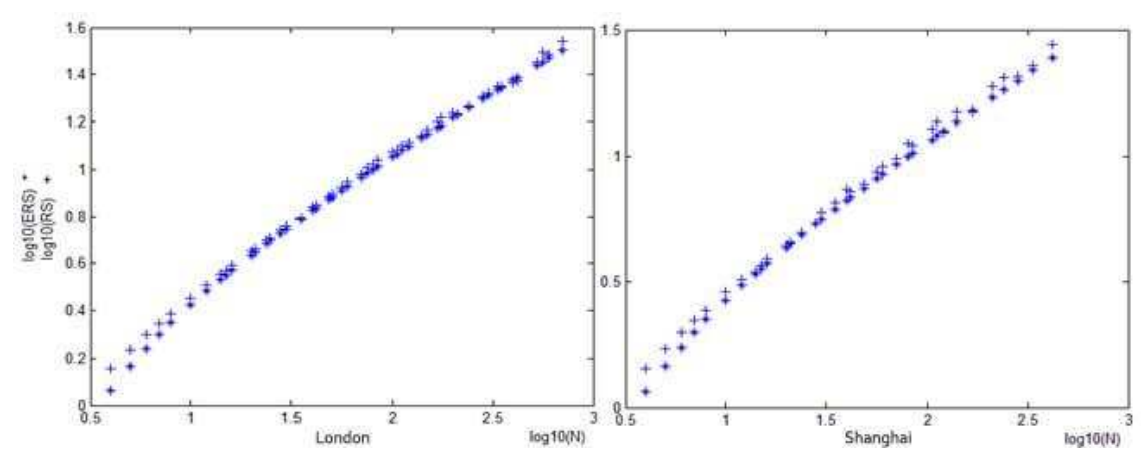

Figure $2 \mathrm{R} / \mathrm{S}$ analysis on fitting model residuals of gold return series in London and Shanghai

\subsection{2 $\mathrm{R} / \mathrm{S}$ contrastive analysis of surrogate data}

To further verify our assumption (i.e. the absense of deterministic process beyond ARMAGARCH in series ${ }^{10}$ ), we generate a simulated ARMA-GARCH process which shares the same parameters with London gold price process ${ }^{11}$. Through $\mathrm{R} / \mathrm{S}$ contrastive analysis and comparison with results of R/S analysis original gold price series in London and Shanghai, we discover

\footnotetext{
${ }^{10}$ Refer to a nonlinear dynamic system that may generate chaos.

${ }^{11}$ Pure ARMA-GARCH process, innovation is simulated by random number.
} 
from Figure 3 that the linear and nonlinear dependence of gold price series in London and Shanghai can be perfectly described by corresponding ARMA-GARCH model. There is little difference between original series and simulated series, suggesting that ARMA-GARCH process can discribe effectively the characteristics of series.

Through R/S test on gold price series, we find the long-term memory existing in original price series rather than in residual series filtered with ARMA-GARCH models. Besides, by simply simulating series with ARMA-GARCH model (same parameters with original series), we can obtain identical characteristics in R/S analysis. This further illustrates that the nonlinear characteristics can be effectively discribed with ARMA-GARCH model, and long-term memory beyond ARMA-GARCH model does not exist in gold price series. The nonlinear characteristic of gold price series is conditional heteroscedasticity, instead of chaos.

\section{Revised Lyapunov exponent analysis}

A system with chaotic structure has an important feature-extreme sensitivity on initial condition, i.e. subtle change in initial value will cause unpredictable enormous variation in the future. The sensitivity can be discribed quantitatively by Lyapunov exponent. Different axis in phase space has different Lyapunov exponent. A negative Lyapunov exponent of a certain axis means that, measured by this axis, two adjacent points tend to approach fixed point and periodic attractors over time, while a positive Lyapunov exponent means the seperation of two adjacent points. This phenomenon is the result of sensitivity on initial condition and thus corresponds with chaotic motion. The zero Lyapunov exponent represents a critical status between stable mode and chaotic mode. It can be viewed either as an end of periodic motion or as the beginning of chaotic motion, but is random in nature.

The study of chaotic characteristics in time series begins with the proposal of theory of reconstructed phase space by Packard et al. in 1980. Due to the nonlinear interaction between all degrees of freedom in the system, the evolution of each independent variable over time contains information about long-term evolution of all other variables in system. As a result, we could study the chaotic behavior of whole nonlinear system through time series of a certain variable. The calculation of Lyapunov exponent based on reconstructed phase space of time series is of significance for determinating whether chaotic behavior exists in a dynamic system that cannot be discribed with differential equation.

\subsection{Reconstructing phase space with C-C algorithm}

To reconstruct phase space, we need two parameters: delay time $T_{d}$ and embedding dimension $m$. Chinese scholar $\mathrm{Lü}^{[9]}$ discovered that, the result of Lyapunov exponent calculation is sensitive to the choice of parameters in reconstruction of phase space. Current methods to choose reconstruction parameters are: methods of autocorrelation, multiple autocorrelation, mutual information and C-C algorithm. To begin with, since the series to be calculated in the paper are residuals of ARMA-GARCH models which have no obvious autocorrelation characteristic, and no smooth and meaningful autocorrelation or multiple autocorrelation curves can be generated, as a result methods of autocorrelation and multiple autocorrelation cannot be used 
in this paper. Besides, due to the large data volumn in this paper ${ }^{12}$, the mutual information method which requires high computation cannot be realized in short period of time.

In an overall consideration of mutual information method's power and computation speed, we choose $\mathrm{C}-\mathrm{C}$ algorithm in this paper. The idea of $\mathrm{C}-\mathrm{C}$ algorithm, as well as BDS tests, is based on correlation integral using the following relationship

$$
S(m, N, r, t)=C(m, N, r, t)-C^{m}(1, N, r, t)
$$

where $r$ stands for correlation radius (same meaning with the proximity parameters in BDS tests). Brock et al. assumed that, given an i.i.d. series, for any $m$ and $r, C(m, N, r, t)$ approximately equals to $C^{m}(1, N, r, t)$ at all time, which can be regarded as a measure of nonlinear dependence. Based on this assumption, we could search for delay time $T_{d}$ by using the figure of $S(m, N, r, t)$ varying with time under nonlinear dependence. The figure can also be viewed as the meaning of changes in autocorrelation function with time under linear dependence.

In order to analyze the nonlinear dependence of series and eliminate short-term spurious correlation, we divide series into $t$ nonintersecting series by time interval $t$, calculate $S\left(m, \frac{N}{t}, r, t\right)$ of each series and obtain the mean. On condition of $t=1$, the divided series is the original series itself

$$
S(m, N, r, 1)=C(m, N, r, 1)-C^{m}(1, N, r, 1)
$$

When $t=2,\left\{X_{i}\right\}$ is divided into $\left\{X_{1}, X_{3}, \cdots, X_{N-1}\right\}$ and $\left\{X_{2}, X_{4}, \cdots, X_{N}\right\}$

$$
S(m, N, r, 2)=\left[C_{1}\left(m, \frac{N}{2}, r, 2\right)-C_{1}^{m}\left(1, \frac{N}{2}, r, 2\right)\right]+\left[C_{2}\left(m, \frac{N}{2}, r, 2\right)-C_{2}^{m}\left(1, \frac{N}{2}, r, 2\right)\right]
$$

For general $t$, the series is divided into $t$ nonintersecting series, having

$$
\bar{S}(m, N, r, t)=\frac{1}{t} \sum\left[C\left(m, \frac{N}{t}, r, t\right)-C^{m}\left(1, \frac{N}{t}, r, t\right)\right]
$$

Suppose that the time series subjects to i.i.d., for a given $m$ and $r$, when $N$ approximates infinite, for all $r$ we have

$$
\bar{S}(m, N, r, t)=0
$$

Although actual series might be quite long, it cannot be treated as infinite, and thus $S(m, N, r, t)$ generally does not equal to zero. As a result, the local largest time interval can be chosen as either null point of $\bar{S}(m, N, r, t)$ or the smallest range time point which is least sensitive to radius change, because the space consists of these points is homogeneous. For convenience, we difine range as follows:

$$
\Delta S(m, N, t)=\max \left(S\left(m, N, R_{i}, t\right)\right)-\min \left(S\left(m, N, R_{j}, t\right)\right)
$$

\subsection{The calculation of Lyapunov exponent}

The major calculation methods of Lyapunov exponent are: defination, Wolf method, Jacobian method, P-norm method and Rosenstein method. To employ defination method we need original differential equations that generate dynamic system, which, unfortunately, we cannot obtain. Wolf method is suitable for small sample and low noise time series, which cannot be

\footnotetext{
${ }^{12}$ The sample size is beyond 10,000 .
} 
adopted in this paper because the sample data (daily trading data over 30 years) are large and noisy. Jacobian method, suitable for large and noisy sample, seems adoptable here, but we find that in this method, Lyapunov exponent is calculated using the speed tangent vector evloves with time, and the tangent vector in reconstructed phase space, which consists of ARMA-GARCH model residuals, shakes violently. As a result, the employment of Jacobian method becomes quite difficult.

In the study of chaos, we can determine a system's chaotic characteristics simply by calculating the largest Lyapunov exponent. Based on that consideration, we choose the Rosenstein method (also called small data set arithmetic), which only calculates the largest Lyapunov exponent. However, traditional Rosenstein method determines delay time $T_{d}$ by autocorrelation method, while here we use C-C algorithm instead. Besides, to employ Rosenstein method we need to determine the average period ${ }^{13}$. Here we choose Fast Foourier Transform.

\subsection{Lyapunov exponents of gold price abroad}

In this paper we use Rosenstein method to calculate largest Lyapunov exponent based on phase space reconstruction by $\mathrm{C}-\mathrm{C}$ algorithm. Although $\mathrm{C}-\mathrm{C}$ algorithm can reduce both the time spent in iterative operation and the space complexity, to determine the real minimal value of $\rho_{S}$, the total length of series has to be as large as possible. For price series in London and New York, we make use of all 9000 samples from ARMA-GARCH model residuals, while for those in Shanghai, the data amount is too limited to search for smallest value of $\rho_{S}$, thus we fail to determine the embedding dimension and reconstruct the phase space. As a result, in this chapter we only listed empirical results of gold data in London and New York.

First we determine the parameters in phase space reconstruction-delay time $T_{d}$ and embedding dimension $m$ (see results Figure 3 and Figure 5). Fig. 4 shows that $\Delta S$ reaches the first minimal value when $T=2$. We choose $T_{d}=2$ as the actual delay for model residual series of London spot. $\rho_{S}$ reaches its minimal value when $t=10$, i.e. $T_{w}=(m-1) T_{d}=10$, from which we obtain embedding dimension $m=6$.
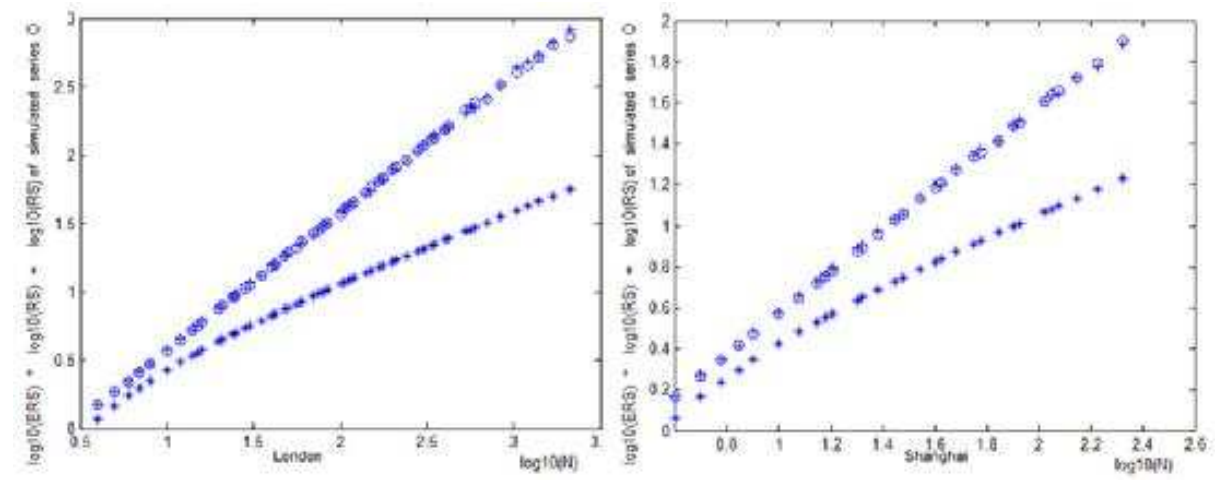

Figure 3 A comparison of R/S analysis on gold original price series in London and Shanghai

\footnotetext{
${ }^{13}$ This average period may not be significant, and the existence of an average period does not necessarily mean the series is periodic. Peters pointed out that the average period of an non-period cycle is just resulted from FFT, which cannot support the series to be periodic.
} 


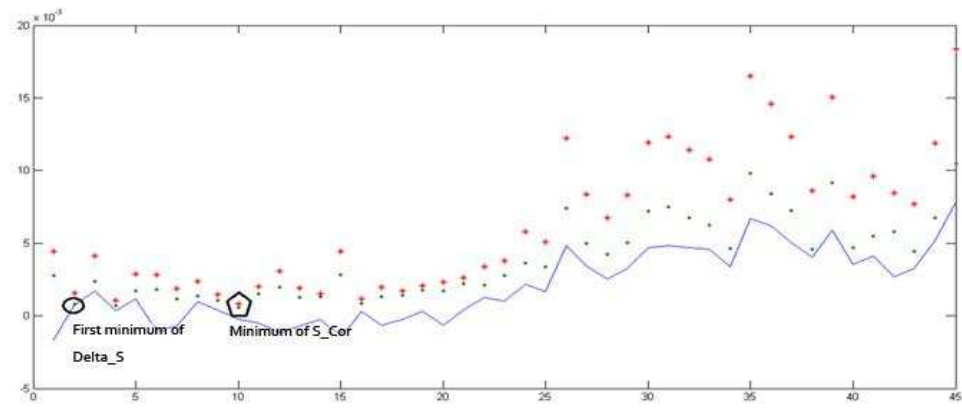

Figure 4 Delay time $T_{d}$ and embedding dimension $m$ for model residuals of London spot

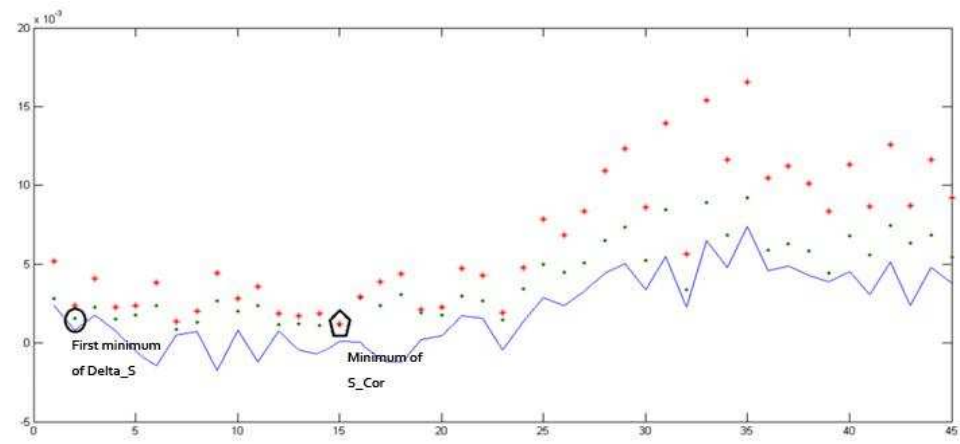

Figure 5 Delay time $T_{d}$ and embedding dimension $m$ for model residuals of New York futures

According to Figure $5, \Delta S$ reaches its first minimal value at $T=2$. As a result, we choose $T_{d}=2$ as actual delay for gold residual series. The minimum of $\rho_{S}$ is reached at $t=15$, i.e. $T_{w}=(m-1) T_{d}=15$ from which we obtain embedding dimension $m=8$.

In this paper we determine the average period with Fast Foourier Transform (FFT), which is a fast algorithm to calculate discrete Foourier Transform. Using this algorithm, the multiplication number required by calculation reduce significantly. The larger the transformed sampling point number $N$ is, the more it saves on calculation cost. By FFT calculation, the average period of fitting model residual series in London and New York is 20.0893 and 34.8231.

Finally, we reconstruct phase space using C-C algorithm, and obtained Lyapunov exponents figure of ARMA-GARCH model residuals with revised Rosenstein method. In Figure 6 (upper one), the slope of line stands for the largest Lyapunov exponents of fitting model residuals in New York. As is obvious, although the slope is negative, it approximates zero $(-5.113574144831425 \mathrm{E}-04)$. This illustrates that fitting model residuals of New York gold futures are random rather than chaotic. According to Figure 6 (lower one) the slope of line is the largest Lyapunov exponent of fitting model residuals in London. From the figure we can clearly see that the slope of the curve approximates zero (0.0062). This illustrates that fitting model residuals of London gold spots are random instead of chaotic.

\section{Conclusion}

In this paper we employed BDS test, rescaled range $(\mathrm{R} / \mathrm{S})$ analysis and revised largest Lyapunov exponents on gold price data at home and abroad, and analyzed the nonlinear attribute 

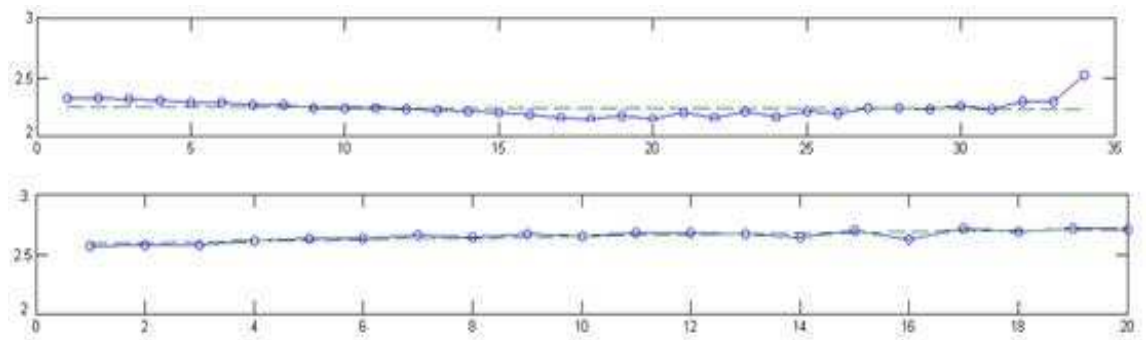

\section{Figure 6 Largest Lyapunov exponents of model residuals in New York (upper) \& London (lower)}

of gold price.

First, we discovered that ARMA model residuals of gold price return series could not pass the BDS test, while standardized residuals of ARMA-GARCH model could pass the BDS test. This suggests that the nonlinear dependence of gold series can be discribed perfectly with GARCH model. Thus, nonlinear dependence beyond ARMA-GARCH model in daily gold price series does not exist, i.e. chaos doesn't exist.

Second, we employed R/S test on original gold price series and discovered strong longterm memory, which did not exist in fitting model residuals. According to the phenomenon that simulated data exhibited same characteristic with original series, we conclude that longterm memory beyond ARMA-GARCH model does not exist in gold price series, and that the nonlinear characteristic of gold price is conditional heteroscedasticity.

Third, we reconstructed phase space according to $\mathrm{C}-\mathrm{C}$ algorithm and discovered that the largest Lyapunov exponent of ARMA-GARCH model residuals approximated zero, which means that chaotic phenomenon, characterized by initial value sensitivity does not exist in fitting model residuals of gold series.

In conclusion, all linear and nonlinear dependence can be effectively discribed by ARMAGARCH model. Long term memory beyond ARMA-GARCH model does not exist in series. Chaotic phenomenon characterized by initial value sensitivity does not exist in fitting model residuals. As a result, the nonlinear characteristic in gold price series is conditional heteroscedasticity instead of $\operatorname{chaos}^{14}$.

\footnotetext{
${ }^{14}$ In order to rule out the possibility that a GARCH model may damage the chaos structure in the original series, we chose a number of typical chaotic system, filtered them with ARMA and GARCH model and then conducted BDS test. The results showed that ARMA and GARCH model did not damage the chaos structure in the series, supporting that ARMA and GARCH model is able to 'safely' filter the characteristics of linearity and time-varying variance out of series. In addition, we analyzed and tested the residuals of 5-minute returns of domestic gold futures, finding out that the standardized residuals of high frequency data model did not follow i.i.d., which means ARMA and GARCH model cannot fully describe the characteristics of high frequency data. However, the largest Lyapunov exponent of the residuals of high frequency data model is -0.03047451402670 , much smaller than that of a daily return series. The negative Lyapunov exponent showed the convergence tendency of the system. More original features were retained in high frequency data of gold futures than in low frequency data, and we suppose this has something to do with the characteristic of mean reversion of futures. Though we chose data of dominant contracts that changed months several times every year, in a period that those contracts did not change, the futures still followed the mean-reverting behavior.
} 


\section{References}

[1] Barnett W A, Gallant A R, Hinich M J, et al. A single-blind controlled competition among tests for nonlinearity and chaos. Journal of Econometrics, 1997, 82(1): 157-192.

[2] Brock W A, Scheinkman J A, Dechert W D, et al. A test for independence based on the correlation dimension. Econometric Reviews, 1996, 15(3): 197-235.

[3] DeCoster G P, Labys W C, Mitchell D W. Evidence of chaos in commodity futures prices. Journal of Futures Markets, 1992, 12(3): 291-305.

[4] Frank M, Stengos T. Measuring the strangeness of gold and silver rates of return. Review of Economic Studies, 1989, 56(4): 553-567.

[5] Grassberger P, Procaccia I. Measuring the strangeness of strange attractors. Physica D: Nonlinear Phenomena, 1983, 9(1): 189-208.

[6] Kanzler L. Very fast and correctly sized estimation of the BDS statistic. Christ Church and Department of Economics, University of Oxford, 1999.

[7] Kim H S, Eykholt R, Salasc J D. Nonlinear dynamics, delay times, and embedding windows. Physica D: Nonlinear Phenomena, 1999, 127(1): 48-60.

[8] Kočenda E, Briatka L'. Optimal range for the iid test based on integration across the correlation integral. Econometric Reviews, 2005, 24(3): 265-296.

[9] Lü J H, Lu J A, Chen S H. Chaotic time series analysis and application. Wuhan University Press, 2002.

[10] Peters E E. Fractal market analysis: Applying chaos theory to investment and economics. John Wiley and Sons, New York, 1994.

[11] Peters E E. Chaos and order in the capital markets: A new view of cycles, prices, and market volatility. John Wiley and Sons, New York, Second edition, 1996.

[12] Rosenstein M T, Collins J J, De Luca C J. A practical method for calculating largest Lyapunov exponents from small data sets. Physica D: Nonlinear Phenomena, 1992, 65(1): 117-134.

[13] Wang W N, Wang B H, Chen X. The statistic analysis of the index of Shanghai securities. Operations Research and Management Science, 2003, 12(4): 85-90.

[14] Xie C, Yang N, Sun B. Empirical research on the chaotic dynamical features of exchange rate time series. Systems Engineering - Theory \& Practice, 2008, 2008(8): 118-122.

[15] Yang S R, Brorsen B W. Nonlinear dynamics of daily futures prices: Conditional heteroskedasticity or chaos? The Journal of Futures Markets, 1993, 13(2): 175-191. 